\title{
Gas-liquid chromatographic analysis of sciatic nerves and spinal cords in experimental allergic neuritis
}

\author{
OTTO APPENZELler, J. MacGEe, AND S. YINGVORAPANT \\ From the Neurology Service and the Medical Research Laboratories of the Veterans' Administration \\ Hospital and the Departments of Neurology, Experimental Medicine and Biological Chemistry, \\ University of Cincinnati College of Medicine, Cincinnati, Ohio, U.S.A.
}

The technique of gas-liquid chromatography is now well-established in the solvent and other industries and is widely applied in biochemical and biomedical research. It offers a number of advantages in the rapid analysis of minute quantities of tissue. Since this technique is capable of separating and detecting substances in microgram quantities it should be particularly useful for the study of samples of tissues where subtle changes are expected. Gas-liquid chromatography generally requires preliminary fractionation and only the fractionated materials are measured. We have employed a technique which gives information about the entire sample without extensive fractionation (MacGee, 1967). The elution curves obtained with this method give 'fingerprints' of the analyzed tissue. Such 'fingerprints' were made from sciatic nerves and cords of controls and animals with experimental allergic neuritis. We also attempted to correlate the changes in the composition of the samples with histological alterations at various stages of the disease.

\section{METHODS}

Random-bred guinea-pigs of both sexes weighing $300-400 \mathrm{~g}$. were obtained from one source. They were kept in single cages on the same rack, fed Purina Chow supplemented by greens and given water ad libitum. The animals were maintained in identical conditions and surroundings until the end of this study two months later.

Sciatic nerves were removed from recently killed guinea-pigs and pooled. They were cleaned of all fat and muscle and were washed repeatedly in normal saline. This tissue was then cut twice on a freezing microtome and emulsified with a pestle and mortar with complete Freund's adjuvant (Difco Laboratories). Injections were made into the toe pads of all four limbs of $0.4 \mathrm{ml}$. of a $25 \%$ emulsion of sciatic nerve in Freund's adjuvant or the same quantity of Freund's adjuvant alone. The animals were examined daily for signs of nervous system disease. Guinea-pigs given sciatic adjuvant were killed at 16 , 26 and 60 days after immunization simultaneously with unimmunized controls and animals injected with Freund's adjuvant alone.

Sciatic nerves and cords were removed from experimental and control animals. One sciatic nerve and part of the lumbar cord from each animal was fixed in formolsaline. Sections for microscopic examination were stained with haematoxylin and eosin. The other sciatic nerves and the remainder of the cord were subjected to gas-liquid chromatography.

The chromatographic unit was a Research Specialties B-600 Series gas chromatograph with an Argon- ${ }^{90} \mathrm{Sr}$ ionization detector and a linear temperature programmer (Warner-Chilcott Laboratories). A $6 \mathrm{ft}$. stainless steel U-tube column packed with $0.5 \%$ benzoylated Carbowax on 80-100 mesh acid-washed glass beads was used. The preparation of the tissues for chromatography is fully described elsewhere (MacGee, 1967). Briefly, an aliquot of $100 \mathrm{mg}$. of wet blotted tissue was heated with $1 \mathrm{ml}$. of tetramethylammonium hydroxide reagent, which completely solubilized it. The digested sample was then evaporated to dryness and, after cooling, extracted with $0.5 \mathrm{ml}$. of ethanol. The relation of sample weight to reagent volume and to the volume of alcohol was kept constant. If less than $100 \mathrm{mg}$. of tissue was available adjustments in the volume of reagent and alcohol were made. These samples were then stored at $-20^{\circ} \mathrm{C}$. until subjected to gas-liquid chromatography.

\section{RESULTS}

Ten guinea-pigs were immunized with sciatic nerve in Freund's adjuvant. Of these, two showed signs of encephalitis 16 days after immunization. This was evident by nystagmus, paralysis, and incontinence. Reluctance to run was observed in three other immunized animals and this was attributed to necrotic ulcers of the foot pads. None of the animals showed clinical signs which were clearly attributable to peripheral neuritis.

Eight guinea-pigs were given Freund's adjuvant alone. In this group there were two with foot pad ulcers which were also reluctant to run. Six guineapigs were kept unimmunized. No spontaneous 
deaths were encountered in these 24 animals over two months until the termination of this study. The results of the histological examinations are given in Table $I$ and in Figures 1, 2, and 3. Dense peri- and epineural mononuclear infiltrates were seen (Fig. 1).

TABLE I

RESULT OF HISTOLOGICAL EXAMINATION OF SCIATIC NERVES AND LUMBAR CORDS IN GUINEA-PIGS IMMUNIZED WITH SCIATIC NERVES IN FREUND'S ADJUVANT

\begin{tabular}{lcccc} 
& $\begin{array}{l}\text { Number with } \\
\text { Disease }\end{array}$ & \multicolumn{3}{c}{ Percentage Severity } \\
\cline { 3 - 5 } & & + & ++ & +++ \\
\hline Nerves & $9 / 10$ & 40 & 60 & 0 \\
Cord & $0 / 10$ & 0 & 0 & 0
\end{tabular}

They were clearly different from the sparse perineural polymorphonuclear cells which have been recognized to represent an agonal change (Waksman and Adams, 1956). Nerves with single perineural lymphocytic aggregates were graded + . Some nerves contained minute foci of mononuclear leucocytes and histiocytes around an endoneural vessel, which were

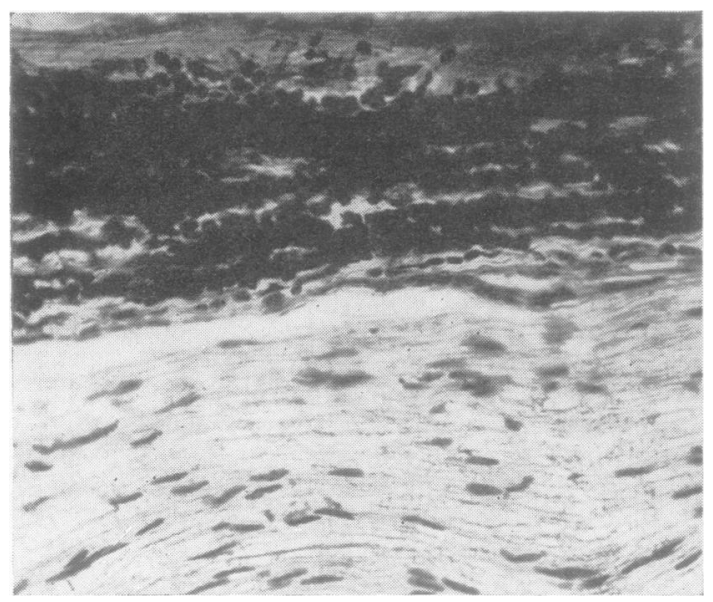

FIG. 1. Longitudinal section of sciatic nerve. Focal dense perivascular aggregation of lymphocytes and mononuclear cells in the epineurium. Animal with experimental allergic neuritis 26 days after inoculation. (Haematoxylin and eosin $\times 316$.)

associated occasionally with degeneration of one or two myelinated fibres (Figs. 2 and 3). If more than two such aggregates were found in the nerve, lesions were graded ++ . In this study large foci of cellular infiltration and myelin destruction were not found. The nerves from animals given Freund's adjuvant alone, or unimmunized controls, showed no recognizable histological lesions. No histological abnormalities were found in the lumbar cords of experimental and control animals.

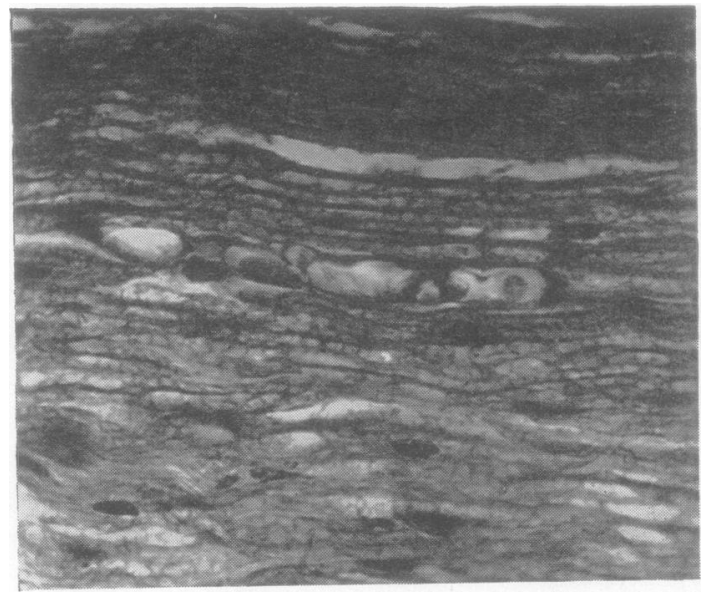

FIG. 2. Longitudinal section of sciatic nerve. A nerve fibre is fragmented into oval masses. The myelin fragments have been removed by solvents during embedding. (Haematoxylin and eosin $\times 504$.)

Gas-liquid chromatograms showed remarkable changes in the 'fingerprints' of nerves from animals with experimental allergic neuritis and those injecteo with Freund's adjuvant alone when compared with healthy controls of similar age. Figures 4 and 5 show curves obtained at 26 days. The area of the 12 mose prominent peaks in the 'fingerprints' of nerves and cords in experimental and control animals was integrated and summated. The percentage of the total area occupied by each of these peaks was the calculated and is given in Tables II and III. The

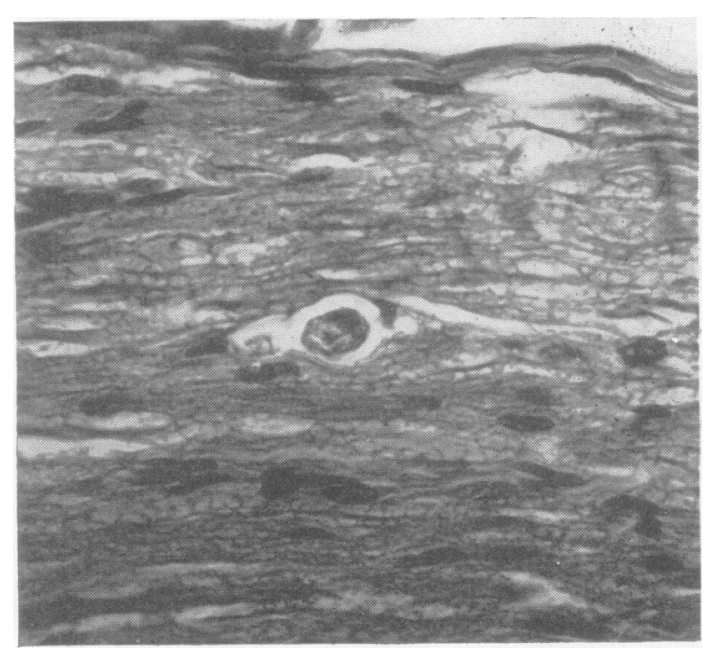

FIG. 3. Same section as in Fig. 2 showing smaller fragmented area of one nerve fibre. (Haematoxylin and eosin $\times 504$.) 
Gas-liquid chromatographic analysis of sciatic nerves and spinal cords in experimental allergic neuritis 281

TABLE II

PERCENTAGE OF TOTAL INTEGRATED AREA FROM GAS-LIQUID CHROMATOGRAM PEAKS OBTAINED FROM GUINEA-PIG SCIATIC NERVES

Peaks $^{1}$

\begin{tabular}{llllllll}
\hline $1-2$ & $3-4$ & 5 & 6 & 7 & $8-9$ & $10-11$ & 12 \\
\hline
\end{tabular}

\begin{tabular}{|c|c|c|c|c|c|c|c|c|}
\hline $\begin{array}{l}16 \text { Days } \\
\text { Normal }\end{array}$ & - & - & - & - & - & - & - & - \\
\hline Freund's adjuvant & 0.8 & $1 \cdot 7$ & 0.4 & $21 \cdot 5$ & $42 \cdot 8$ & $11 \cdot 7$ & $1 \cdot 7$ & $19 \cdot 4$ \\
\hline Freund's + sciatic nerve & 0.7 & 1.0 & $0 \cdot 7$ & $25 \cdot 4$ & $40 \cdot 7$ & $23 \cdot 5$ & 1.5 & 65 \\
\hline $\begin{array}{l}26 \text { Days } \\
\text { Normal } \\
\text { Freund's adjuvant } \\
\text { Freund's + sciatic nerve }\end{array}$ & $\begin{array}{l}0.6 \\
0.6 \\
0.6\end{array}$ & $\begin{array}{l}1.4 \\
1.5 \\
1.8\end{array}$ & $\begin{array}{l}0.6 \\
1 \cdot 1 \\
0.4\end{array}$ & $\begin{array}{l}26 \cdot 7 \\
32 \cdot 5 \\
25 \cdot 2\end{array}$ & $\begin{array}{l}47 \cdot 9 \\
39 \cdot 3 \\
36 \cdot 8\end{array}$ & $\begin{array}{l}11.4 \\
20.4 \\
12.4\end{array}$ & $\begin{array}{l}1 \cdot 0 \\
1 \cdot 5 \\
1 \cdot 1\end{array}$ & $\begin{array}{r}10 \cdot 3 \\
3.2 \\
21.5\end{array}$ \\
\hline $\begin{array}{l}60 \text { Days } \\
\text { Normal } \\
\text { Freund's adjuvant } \\
\text { Freund's + sciatic nerve }\end{array}$ & $\begin{array}{l}0.7 \\
0.4 \\
0.5\end{array}$ & $\begin{array}{l}1.4 \\
1.3 \\
1.2\end{array}$ & $\begin{array}{l}0.9 \\
0.7 \\
0.8\end{array}$ & $\begin{array}{l}30 \cdot 6 \\
28 \cdot 2 \\
29 \cdot 0\end{array}$ & $\begin{array}{l}38 \cdot 4 \\
38 \cdot 0 \\
36 \cdot 5\end{array}$ & $\begin{array}{l}20 \cdot 7 \\
17 \cdot 6 \\
19 \cdot 9\end{array}$ & $\begin{array}{l}1 \cdot 7 \\
2 \cdot 3 \\
2 \cdot 4\end{array}$ & $\begin{array}{r}6.4 \\
11.6 \\
9.7\end{array}$ \\
\hline
\end{tabular}

${ }^{1}$ Peaks $1-2 ; 3-4 ; 8-9$ and $10-11$ each were considered as one peak in the calculations.

TABLE III

PERCENTAGE OF TOTAL INTEGRATED AREA FROM GAS-LIQUID CHROMATOGRAM PEAKS OBTAINED FROM GUINEA-PIG LUMBAR CORDS

\begin{tabular}{|c|c|c|c|c|c|c|c|c|c|c|}
\hline & \multicolumn{10}{|c|}{ Peaks $^{1}$} \\
\hline & $1-2$ & 3 & 4 & 5 & 6 & 7 & 8 & 9 & $10-11$ & 12 \\
\hline \multicolumn{11}{|l|}{16 Days } \\
\hline Freund's adjuvant & $\mathbf{0}$ & $\mathbf{0}$ & $2 \cdot 1$ & 0 & $10 \cdot 1$ & $32 \cdot 8$ & 0 & $4 \cdot 1$ & $5 \cdot 7$ & $45 \cdot 2$ \\
\hline Freund's + sciatic nerve & 0 & 0 & $3 \cdot 6$ & 0 & $11 \cdot 7$ & $33 \cdot 7$ & 0 & $3 \cdot 4$ & $4 \cdot 1$ & $43 \cdot \overline{4}$ \\
\hline \multicolumn{11}{|l|}{26 Days } \\
\hline Normal & $\mathbf{0}$ & 0 & $2 \cdot 6$ & 0 & $7 \cdot 6$ & $29 \cdot 7$ & 0 . & $4 \cdot 7$ & 3.9 & $51 \cdot 4$ \\
\hline Freund's adjuvant & $\mathbf{0}$ & 0 & $2 \cdot 0$ & 0 & 7.6 & $29 \cdot 5$ & 0 & $5 \cdot 3$ & $4 \cdot 6$ & $51 \cdot 0$ \\
\hline Freund's + sciatic nerve & 0 & 0 & $2 \cdot 9$ & 0 & $5 \cdot 3$ & $31 \cdot 2$ & 0 & $4 \cdot 3$ & $4 \cdot 5$ & 51.8 \\
\hline \multicolumn{11}{|l|}{60 Days } \\
\hline Normal & $\mathbf{0}$ & 0 & $2 \cdot 0$ & $\mathbf{0}$ & $10 \cdot 0$ & $27 \cdot 8$ & 0 & $5 \cdot 3$ & 3.9 & $51 \cdot 1$ \\
\hline Freund's adjuvant & 0 & 0 & $1 \cdot 8$ & 0 & 7.0 & $30 \cdot 4$ & $\mathbf{0}$ & $5 \cdot 0$ & $4 \cdot 4$ & $51 \cdot 4$ \\
\hline Freund's + sciatic nerve & 0 & 0 & $1 \cdot 7$ & 0 & $7 \cdot 8$ & $30 \cdot 0$ & 0 & $5 \cdot 8$ & $4 \cdot 3$ & $50 \cdot 7$ \\
\hline
\end{tabular}

${ }^{1}$ Peaks 10-11 were considered as one peak in the calculations.

percentage distribution of the peaks in the chromatograms of nerves of unimmunized animals showed changes with advancing age. Guinea-pig nerves from animals given Freund's adjuvant alone showed a remarkable increase in peaks nos. 5, 8-9 and a corresponding fall in peak no. 12 at 26 days. Sixty days after immunization there was a return toward the original level (Table II).

The nerves from animals with experimental allergic neuritis showed an increase in peaks nos. 8-9 at 16 days and falls in peaks 3-4 and 12, whereas at 26 days there was a marked increase in peak no. 12 (Fig. 4). At the end of this study the peaks did not differ appreciably from those obtained from unimmunized animals kept for a similar periods of time (Table II). No significant changes occurred in chromatograms prepared from spinal cord at the same period (Fig. 5).

A tentative identification of the substances responsible for peaks 1-12 was made by comparing their retention times with those obtained from pure fatty acids and cholesterol which had been subjected
TABLE IV

TENTATIVE IDENTIFICATION OF PEAKS 1-12 IN GAS-LIQUID CHROMATOGRAMS

\begin{tabular}{lll}
$\begin{array}{l}\text { Peak } \\
\text { No. }\end{array}$ & Possible Fatty Acid & No. C:Double Bonds \\
\hline 1 & & \\
2 & Caprylic & $8: 0$ \\
3 & & \\
4 & & \\
5 & & $14: 0$ \\
6 & Myristic & $16: 0$ \\
7 & Palmitic & $18: 0+18: 1+18: 3$ \\
8 & Stearic + oleic + linolenic & $18: 2+18: 3$ \\
9 & Linoleic + linolenic & $18: 2+18: 3$ \\
10 & Linoleic + linolenic & $18: 2+18: 3$ \\
11 & Linoleic + linolenic & $22: 0$ \\
12 & Behenic &
\end{tabular}

${ }^{1}$ The assignment of more than one position to linoleic acid (18:2) and linolenic acid $(18: 3)$ is a result of the isomerization and fragmentation of the poly-unsaturated acids by the strong alkali and heat treatments given.

to the same digestion procedures. Table IV lists the substances with retention times similar to peaks $1-12$.

Correlation of changes in the composition of the 


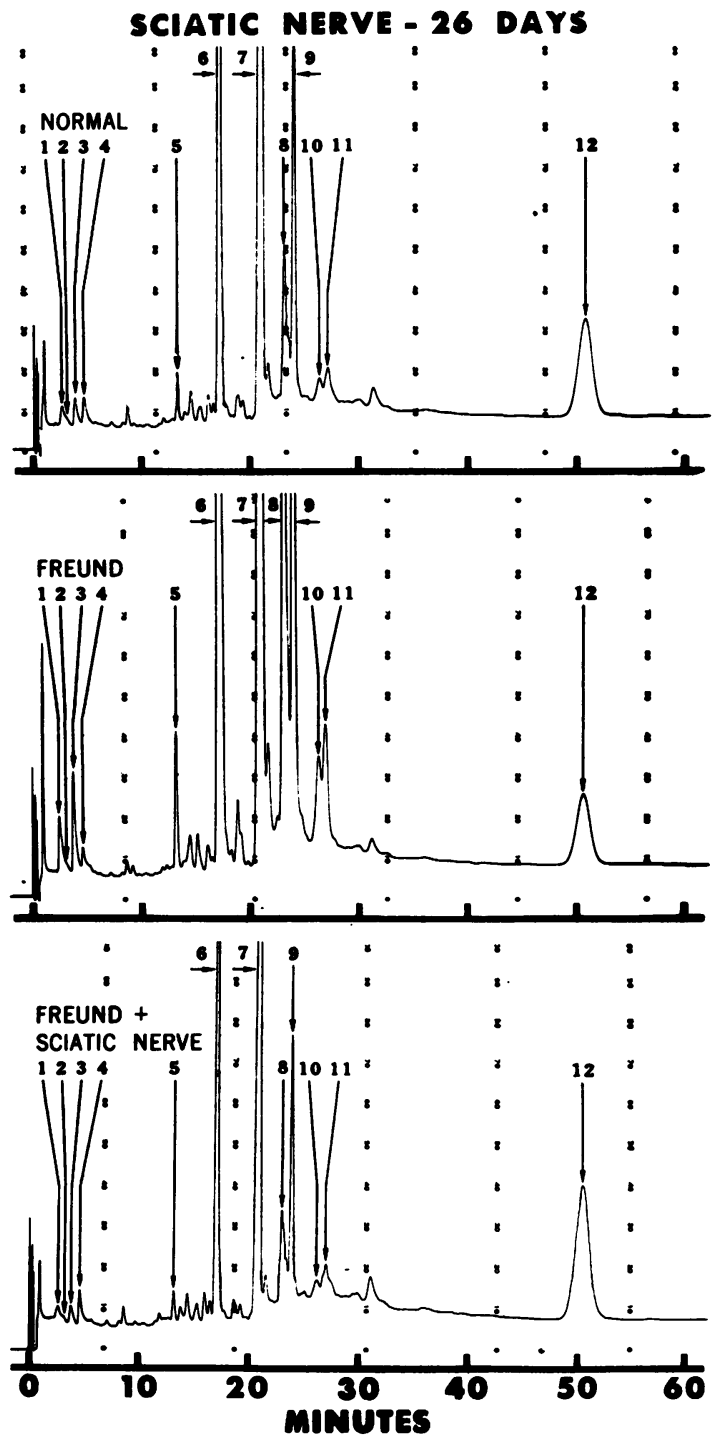

FIG. 4.

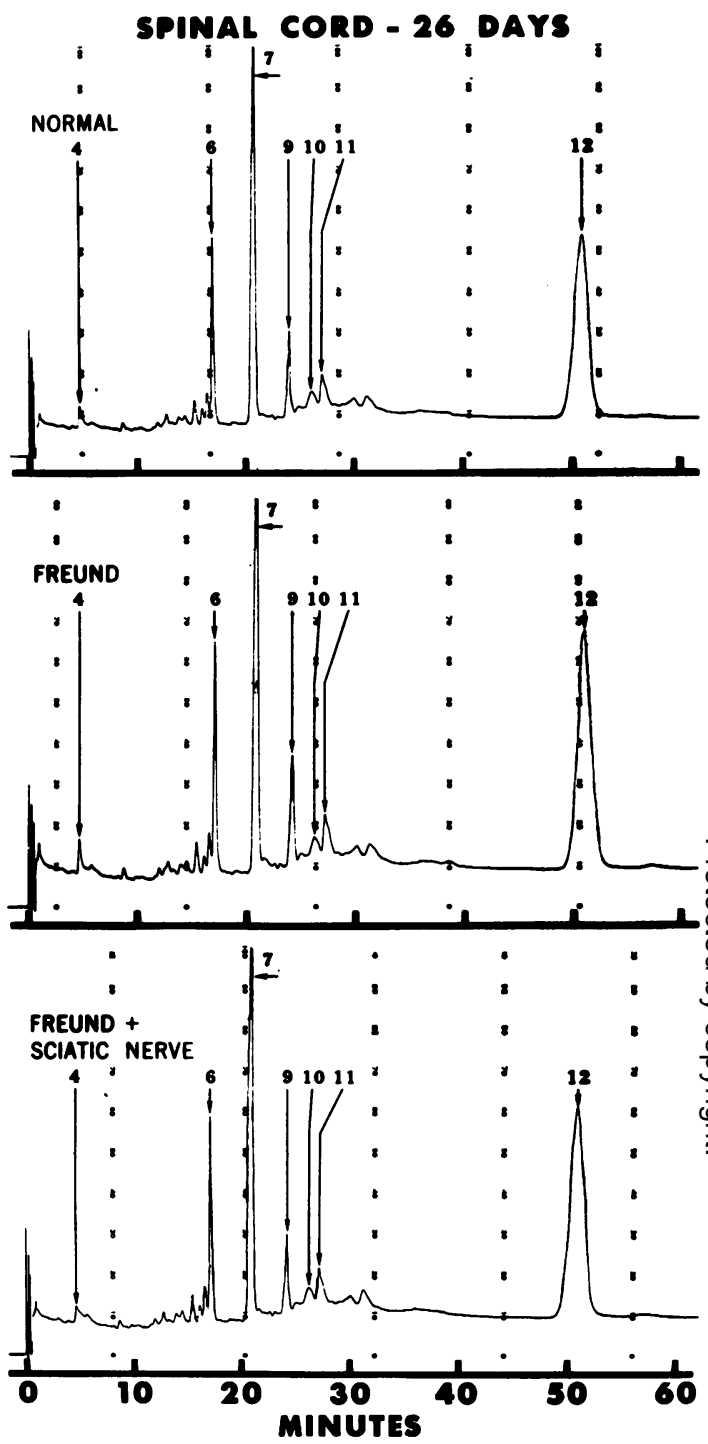

FIG. 5.

FIG. 4. Gas-liquid chromatographic elution patterns ('fingerprints') of guinea-pig sciatic nerves. Controls (normal and Freund) and animals with experimental allergic neuritis (Freund + sciatic nerve) at 26 days. The numbers of the peaks correspond to those listed in Table IV.

FIG. 5. Gas-liquid chromatographic elution patterns ('fingerprints') of guinea-pig lumbar cords at 26 days. The labels and numbers are similar to those in Figure 4.

nerves with the histological lesions seen at various stages of experimental allergic neuritis was unsuccessful. Many nerves were histologically normal and yet they showed marked alteration in their chemical composition.

\section{DISCUSSION}

Experimental allergic neuritis can be induced in a variety of species but in the guinea-pig the correlation of clinical signs with peripheral nerve 
lesions was poor (Waksman and Adams, 1956). Moreover, when guinea-pig sciatic nerves were used as the antigenic stimulus lesions in both central and peripheral nervous systems were produced. No guinea-pig with peripheral nerve lesions alone was found. In animals with affected nerves focal and connective tissue lesions were seen but Wallerian type of change was rare (Waksman and Adams, 1956). The assumption has often been made that changes in the composition of nerves may precede histological alteration or even be the only change associated with some peripheral neuropathies. Because of this we selected the guinea-pig and immunization procedures used in this study, since our main purpose was to try and delineate the biochemical changes of experimental allergic neuritis. In unimmunized animals analysis of peripheral nerves by gas-liquid chromatography showed alterations in their composition with advancing age. This is not surprising, since in the few morphological studies of human nerves, changes in fibre count and distribution of fibre size were also found in old age (Swallow, 1966).

The analysis of tissue by gas-liquid chromatography allowed us to recognize profound biochemical changes without clinical signs of neuritis and with minimal or no histological alterations in the nerves. The correlation with focal and connective tissue lesions was also poor, and changes were detected in histologically normal nerves from animals immunized with sciatic antigen.

In this study the lumbar cords showed no lesions and there were no alterations in the composition of the samples. In experimental allergic neuritis induced by peripheral nerve more than half of the guinea-pigs had only mild lesions when a histological survey of the entire central nervous system was made (Waksman and Adams, 1956). The absence of histological and biochemical lesions in the cord may therefore be attributed to the focal nature of the disease in the central nervous system and restricted sample examined in this study.

A surprising finding was the biochemical changes in peripheral nerves produced by the injection of Freund's adjuvant alone which were evident at 26 days and disappeared 60 days after injection. The return to normality after two months suggests that they were indeed produced by the adjuvant injection and were clearly recognizable in the nerves but not in the cords of these animals. Laufer, Tal, and Behar (1959) examined histological changes in guinea-pigs produced by the injection of complete Freund's adjuvant and its various fractions. They found proliferation of reticular endothelial cells in the liver and red pulp cells in the spleen. No reaction whatever was elicited in the brain, heart, or kidneys but peripheral nerves were not examined. In our histologically examined peripheral nerves no reaction to Freund's adjuvant was seen but biochemical changes were detectable by gas-liquid chromatography. It has been suggested that isoimmune lesions in rabbits, hamsters, and guinea-pigs should be interpreted with caution whenever Freund's adjuvant is used for potentiation of tissue antigens. The metastatic localization of granulomata to various organs other than the nervous system has been attributed, at least in part, to lymphatic and haematogenous dissemination of the adjuvant (Steiner, Langer, and Schatz, 1960). For this reason we subjected our Freund's adjuvant to gas-liquid chromatography and found in it only one major peak (no. 7). The changes in the peripheral nerves induced by its injection, however, were predominantly in peaks 5, 8-9 and 12 , suggesting that this was not due to a simple spread of adjuvant from the injection site to the nerves. It would seem, therefore, that the injection of Freund's adjuvant in guineapigs is liable to alter the nervous system and that such animals are not well chosen as the only controls for studies of experimental diseases of the peripheral nerves.

The lipid class and fatty acid composition of cat and chicken peripheral nerve during Wallerian degeneration has been studied by Berry, Cevallos, and Wade (1965). It was found that all fatty acids were markedly decreased 90 days after section. Triglycerides decreased after eight days, returning to normal by 96 days after cutting, accompanied by a transient increase in free fatty acids. Cholesterol esters increased markedly at eight days and remained higher than normal 96 days after section. We observed less drastic changes in experimental allergic neuritis. In general, there was a reciprocal relation between the percentage of cholesterol and fatty acid peaks at various stages of the disorder. Cholesterol fell but the fatty acids showed a relative increase early and the reverse happened later in the disease. Sixty days after immunization the proportion of various peaks returned toward normal. In our experimental material definite Wallerian degeneration was not observed and damage to myelin sheaths, as judged from histological examination, was minor, yet the biochemical changes evident on the 'fingerprints' were clearly distinguishable from controls.

The observations are of more than academic interest and may lead to improvement in our understanding of human peripheral neuropathies. It is generally known that symptoms and signs of peripheral nerve dysfunction may be severe without recognizable histological lesions, even after extensive sampling and expert examination at necropsy. 
Since gas-liquid chromatography is capable of analysing small samples and rapidly gives a 'fingerprint' of tissue obtained by biopsy, it may prove a reliable method for the detection of specific biochemical changes in nerves from patients with neuropathies.

\section{SUMMARY}

Sciatic nerves and lumbar cords were studied histologically and examined by gas-liquid chromatography in experimental allergic neuritis in the guinea-pig. No histological or biochemical alterations were found in the cords of experimental and control animals. In experimental animals there were no clinical signs of neuritis and no correlation was found between lesions in nerves and biochemical changes. Many histologically normal nerves showed profound biochemical changes induced by the experimental procedures. In sciatic nerves the histological lesions were mild. Biochemical alterations were noted in unimmunized controls with advancing age and were also induced by Freund's adjuvant. It is suggested that injection of Freund's adjuvant affects the composition of peripheral nerves and that such animals are not suitable as the only controls for the study of experimental neuropathies. Cholesterol and fatty acid composition showed a reciprocal relationship. A fall in the former and relative increase in the latter were observed early in the disease and the reverse was seen 26 days after immunization. By 60 days the composition of peripheral nerves in experimental animals resembled that of unimmunized controls kept for a similar length of time. It is suggested that the examination of peripheral nerves by gas-liquid chromatography gives a better indication of disease than histological survey and may prove useful in the examination of human biopsy material.

The excellent technical assistance of Mr. Kenneth G Allen is acknowledged.

\section{REFERENCES}

Berry, J. F., Cevallos, W. H., and Wade, R.R., Jr. (1965). Lipid class and fatty acid composition of intact peripheral nerve and during Wallerian degeneration. J. Amer. oil chem. Soc., 42, 492-500.

Laufer, A., Tal, C., and Behar, A. J. (1959). Effect of adjuvant (Freund's type) and its components on the organs of various animal species. A comparative study. Brit. J. exp. Path., 40, 1-7.

MacGee, J. (1967). Characterization of mammalian tissue and microorganisms by gas-liquid chromatography. J. Gas Chromatog., Z 5 (in press).

Steiner, J. W., Langer, B., and Schatz, D. L. (1960). The local and $\mathcal{O}$

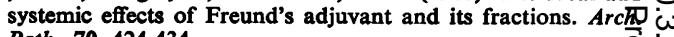
Path., 70, 424-434.

Swallow, M. (1966). Fibre size and content of the anterior tibial nervi of the foot. J. Neurol. Neurosurg. Psychiat., 29, 205-213.

Waksman, B. H., and Adams, R. D. (1956). A comparative study 8 음 experimental allergic neuritis in the rabbit, guinea-pig and mouse. J. Neuropath. exp. Neurol., 15, 293-333. 